\title{
Average Case Analysis of the MST-heuristic for the Power Assignment Problem: Special Cases
}

\author{
Maurits de Graaf \\ Thales Nederland B.V., University of Twente \\ P.O. Box 217 \\ 7500 AE Enschede, Netherlands \\ M.deGraaf@utwente.nl
}

\author{
Richard J. Boucherie, Johann L. Hurink, \\ and Jan-Kees van Ommeren \\ University of Twente \\ P.O. Box 217 \\ 7500 AE Enschede, Netherlands \\ $\{$ R.J.Boucherie, J.L.Hurink, \\ J.C.W.vanOmmeren\}@utwente.nl
}

\begin{abstract}
We present an average case analysis of the minimum spanning tree heuristic for the power assignment problem. The worst-case approximation ratio of this heuristic is 2 . We have the following results: (a) In the one-dimensional case, with uniform $[0,1]$-distributed distances, the expected approximation ratio is bounded above by $2-2 /(p+2)$, where $p$ denotes the distance power gradient. (b) For the complete graph, with uniform $[0,1]$ distributed edge weights, the expected approximation ratio is bounded above by $2-1 / 2 \zeta(3)$, where $\zeta$ denotes the Riemann zeta function.
\end{abstract}

\section{Categories and Subject Descriptors}

G2.2.2 [Discrete Mathematics]: Graph Theory

\section{General Terms}

Theory

\section{Keywords}

power assignment, minimum spanning tree, random graphs

\section{INTRODUCTION}

Ad hoc wireless networks have received significant attention due to their potential applications (see, for example [10]). In such a network, communication takes place either through single-hop transmission, or by relaying through intermediate nodes. The topology (the set of communication links) depends on uncontrollable factors (node mobility) and on controllable parameters (transmit power). We assume an idealized propagation model, with omnidirectional transceivers with adjustable transmit power. For assigning transmit powers, two conflicting effects have to be taken into account: if transmit powers are too low, the resulting topology may be too sparse. On the other extreme, if transmit powers are too high, the nodes run out of energy quickly.

The goal of the Connected Minimum Power Assignment (CMPA-) problem is to assign transmit powers such that the resulting network is connected and the sum of transmit powers is minimized (see e.g. [10]). This problem is, in general, NP-hard (for some special cases there are polynomial solutions). The intuitive MST-heuristic is known to have a worst-case approximation ratio of 2 . This paper analyses the average-case approximation ratio. A directional variant of this problem, the Minimum Energy Broadcast Routing (MEBR) problem is studied in [3]. A related numerical study is carried out in [11]. The results of this work can be used in assessing whether, in a concrete network, a given power assignment can be further optimized.

\subsection{Notation, related work and contribution}

For a set of points $V$ representing the nodes in a network, a power assignment can be represented as a function $\mathbf{p}: V \rightarrow$ $\mathbb{R}_{0}^{+}$. Following the notation of [10], for each ordered pair $(u, v)$ of transceivers, there is a transmit power threshold, denoted by $c(u, v)$, with the following meaning: a signal transmitted by the transceiver $u$ can be received by $v$ only when the transmit power $\mathbf{p}(u)$ is at least $c(u, v)$. We assume that for each pair of points the values $c(u, v)$ are known and symmetric, i.e., $c(u, v)=c(v, u)$ for all pairs $\{u, v\} \in V$. A power assignment $\mathbf{p}$ defines an undirected graph $G_{\mathbf{p}}=$ $\left(V, E_{\mathbf{p}}\right)$, where $e=\{u, v\} \in E_{\mathbf{p}}$ if and only if $\mathbf{p}(u) \geq c(u, v)$ and $\mathbf{p}(v) \geq c(u, v)$. Note that in the case $\mathbf{p}(v) \geq c(u, v)>$ $\mathbf{p}(u)$ only transmission from $v$ to $u$ is possible. However, in this paper only symmetric links are considered.

This paper deals with the CMPA-problem: given a graph $G=(V, E, c)$, where $c$ denotes the edge weights $c: E \rightarrow \mathbb{R}^{+}$, one asks for a power assignment $\mathbf{p}: V \rightarrow \mathbb{R}_{0}^{+}$such that $G_{\mathbf{p}}$ is connected and the total power $\sum_{v \in V} \mathbf{p}(v)$ is minimal.

When $V \subset \mathbb{R}^{d}$, a power attenuation model is assumed, assuming that the signal power decreases with the distance $r$ as $r^{-p}$, where the distance-power gradient $p \in \mathbb{R}^{+}$depends on the wireless environment. This implies that $c(u, v)=r^{p}$ if the distance between $u$ and $v$ is $r$. Therefore, in this case, the power assignment problem corresponds to assigning a range $r_{v}$ to node $v$. This is called the range assignment problem.

The range assignment problem is NP-hard in all dimensions $d \geq 2$ for all values of the distance-power gradient $p$ [5]. Based on these complexity results, polynomial time approximation algorithms were studied. The first approximation 
algorithm to the CMPA-problem is the Minimum Spanning Tree (MST)-algorithm (see [4], [7]). This work complements [8] and [9], in providing a rigorous proof for the general case with uniform edge weights (Section 4 of this paper), and in analysing the (more complex) MST-heuristic instead of the MST-functional.

MST Power Assignment Algorithm ( $V, E, c)$ : (1) Compute a minimum spanning tree $T$ using $c$ as edge costs; (2) For each node $v \in V$ assign

$$
\mathbf{p}(v)=\max \{c(e) \mid e \text { incident to } v \text { in } T\} .
$$

Let $T_{n}$ denote a minimum spanning tree of a graph on $n$ vertices. In addition, let $P_{n}$ denote the power assignment corresponding to $T_{n}$, i.e., for each $v \in V: P_{n}(v)=$ $\max \left\{c(e) \mid e \in T_{n}\right.$ and $e$ incident to $\left.v\right\}$. We define $W\left(T_{n}\right)$ to be the total weight of the spanning tree $T_{n}$, and $W\left(P_{n}\right)$ the total power of the corresponding power assignment. It is well established (see e.g. [1], [4]) that

$$
W\left(T_{n}\right) \leq W(P) \leq W\left(P_{n}\right) \leq 2 W\left(T_{n}\right)
$$

where $W(P)$ denotes the total power of the optimal power assignment $P$. In [1] it is shown that the factor 2 is tight.

For the MST algorithm, (1) shows that the worst-case performance ratio is 2 . Other approximation algorithms are studied in [1], where a polynomial time approximation scheme with a worst-case performance ratio approaching $5 / 3$ as well as a more practical approximation algorithm with a worst-case approximation factor of $11 / 6$ are given.

While the worst-case performance ratio of 2 might discourage use of the MST algorithm in practice, numerical results indicate that the MST algorithm is often rather close to the optimal solution [12]. This motivates an average case analysis.

Statement of contribution. This paper presents an analysis of the function $W\left(P_{n}\right) / W\left(T_{n}\right)$, in the average case, for $n \rightarrow \infty$, which provides a general upper bound to the average case performance ratio $W\left(P_{n}\right) / W(P)$. Here, we investigate the 1-dimensional situation, as a stepping stone for similar analysis in higer dimensions. Then, we investigate the situation for the complete graph with independent uniform edge weights, where the expected approximation ratio turns out to have a closed form upper bound well below the worst-case upper bound.

The paper is organized as follows. Section 2 provides preliminary results. Section 3 analyzes the 1-dimensional case. Section 4 shows a result on the average case performance for complete graphs with independent uniformly distributed $[0,1]$ edge weights. Finally, Section 5 presents concluding remarks.

\section{PRELIMINARIES ON MINIMUM SPAN- NING TREES}

Let $G=(V, E)$ be a graph with $|V|=n$ and a cost function $c: E \rightarrow \mathbb{R}^{+}$. Furthermore, for a vertex $v$, let $G \backslash\{v\}$ denote the graph arising from $G$ by deleting $v$ and all edges incident to $v$, for an edge $e, G \backslash\{e\}$ denotes the graph arising from $G$ by deleting edge $e$. Suppose $F=\left(V, E_{F}\right)$ is a forest on $G$ (i.e., a graph with no cycles) with $E_{F}=$ $\left\{e_{1}, \ldots, e_{m}\right\} \subset E$. We assume $c\left(e_{1}\right) \leq c\left(e_{2}\right) \leq \ldots \leq c\left(e_{m}\right)$ and let $S_{k}(F)=\sum_{j=m-k+1}^{m} c\left(e_{j}\right)$ denote the sum of the $k$ heaviest edges of $F$, for $k \in\{1, \ldots, m\}$. If $m=n-1$, then
$F$ is a spanning tree, which we denote by $T$. For a given tree $T$, we say that an edge $e$ incident to $v$ covers $v$, if (a) $e \in T$, i.e., $e=e_{i}$ for some $i \in\{1, \ldots, n-1\}$, and (b) the index $i$ is maximal among the edges $e$ incident to $v$. Note that condition (b) ensures that each vertex is covered by exactly one edge and that $c\left(e_{i}\right) \geq c\left(e_{j}\right)$ for all edges $e_{j} \in T$ incident to $v$.

Let $T_{n}$ be a minimum spanning tree and let $f(e)$ denote the number of nodes covered by $e \in T_{n}$, called the covering number of $e \in E$. Note that $f(e) \in\{0,1,2\}$. We immediately see that $\sum_{e \in E} f(e)=n$ as each vertex is covered exactly once. Moreover, $W\left(P_{n}\right)=\sum_{e \in T_{n}} f(e) c(e)$.

The following observation strengthens $(1)$.

Lemma 1. Let the edges $e_{1}, \ldots, e_{n-1}$ of a minimum spanning tree $T_{n}$ be sorted such that $c\left(e_{1}\right) \leq \ldots \leq c\left(e_{n-1}\right)$. Then

$$
c\left(e_{n-1}\right)+S_{n-1}\left(T_{n}\right) \leq W\left(P_{n}\right)
$$

and,

$$
W\left(P_{n}\right) \leq \begin{cases}c\left(e_{\lfloor n / 2\rfloor}\right)+2 S_{\lfloor n / 2\rfloor}\left(T_{n}\right) & \text { if } n \text { is odd, } \\ 2 S_{n / 2}\left(T_{n}\right) & \text { if } n \text { is even. }\end{cases}
$$

Proof. Inequality (2) can be inferred by induction on $n$, using the fact that each tree contains at least 2 vertices of degree 1. The inequalities of (3) follow from the fact that $f(e) \in\{0,1,2\}$ and $\sum_{e \in E} f(e)=n$. Therefore $W\left(P_{n}\right)=$ $\sum_{i=1}^{n-1} f\left(e_{i}\right) c\left(e_{i}\right)$ takes its maximum, when $f$ takes maximum values for the edges with the highest weights.

The following example shows that the bounds for the inequalities (2) and (3) are tight.

ExAmPle 1. Let $G=(V, E)$ be a path $e_{1}, \ldots, e_{n}$ such that $c\left(e_{j}\right)=1$ if $j$ is odd, and $c\left(e_{j}\right)=\epsilon<1$ if $j$ is even. $G$ has only one spanning tree $T$ which is equal to the graph itself: $T=G$. Sorting the edges according to increasing costs we first obtain $\lceil(n-1) / 2\rceil$ edges of cost $\epsilon$, followed by $\lfloor(n-1) / 2\rfloor$ edges of cost 1 . Moreover, $W(T)=\lceil(n-$ $1) / 2\rceil+\lfloor(n-1) / 2\rfloor \epsilon$. Clearly, all edges with an odd index have covering number 2, and, if $n$ is odd, say $n=2 m+1$, there is only one edge (being $e_{2 m}$ ) with covering number 1 , incident to the last vertex. So $W\left(P_{T}\right)=2 m+\epsilon$, which is a tight bound for the right hand side of (3). If $n$ is even, say $n=2 m$ then $W\left(P_{T}\right)=2 m$, which is a tight bound for the right hand side of (3). An example for tightness of the left hand side of (3) is obtained by considering a graph $G=(V, E)$ where all costs $c(e)$ are 1 . In this case $W(T)=n-1$ and $W\left(P_{T}\right)=n$.

Lemma 1 implies,

$$
W\left(P_{n}\right) \leq 2 S_{\lceil n / 2\rceil}\left(T_{n}\right) .
$$

\section{ONE DIMENSION: SPANNING TREE IS PATH}

We consider the situation where $G=(V, E)$ is a path of length $n, X_{1} \leq \ldots \leq X_{n+1} \in \mathbb{R}^{1}$ where the transmit power thresholds $D_{i}=X_{i+1}-X_{i}$ to connect neighboring vertices are i.i.d. nonnegative random variables with finite expectation.

TheOREm 1. Let $G=(V, E)$ be a path as defined above. Then

$$
\frac{W\left(P_{n}\right)}{W\left(T_{n}\right)} \stackrel{a . s .}{\longrightarrow} \frac{\mathbb{E}\left[\max \left\{D_{1}, D_{2}\right\}\right]}{\mathbb{E}\left[D_{1}\right]} .
$$


Proof. First we split the sum in odd and even terms:

$$
\begin{aligned}
W\left(P_{n}\right) & =D_{1}+\sum_{i=1}^{n-1} \max \left\{D_{i}, D_{i+1}\right\}+D_{n} \\
& =D_{1}+\sum_{i=1}^{\lfloor n / 2\rfloor} \max \left\{D_{2 i-1}, D_{2 i}\right\} \\
& +\sum_{i=1}^{\lfloor(n-1) / 2\rfloor} \max \left\{D_{2 i}, D_{2 i+1}\right\}+D_{n} .
\end{aligned}
$$

Since, by splitting the odd and even terms, in both sums the random variables are i.i.d., it follows by the strong law of large numbers, that

$$
\frac{W\left(P_{n}\right)}{n} \stackrel{a . s}{\longrightarrow} \mathbb{E}\left[\max \left\{D_{1}, D_{2}\right\}\right] .
$$

Being the sum of i.i.d. r.v.'s, $W\left(T_{n}\right)$ also satisfies the strong law of large numbers, and we obtain:

$$
\frac{W\left(T_{n}\right)}{n} \stackrel{a . s}{\longrightarrow} E\left[D_{1}\right]
$$

implying almost sure convergence of $\frac{W\left(P_{n}\right)}{W\left(T_{n}\right)}$.

Since, $W\left(P_{n}\right)$ and $W\left(T_{n}\right)$ are sequences of r.v.'s, with bounded ratio, the above theorem implies that these ratios also converge in mean and that $\mathbb{E}\left[W\left(P_{n}\right) / W\left(T_{n}\right)\right]$ converges. So we have:

\section{Corrollary 1.}

$$
\lim _{n \rightarrow \infty} \mathbb{E}\left[\frac{W\left(P_{n}\right)}{W\left(T_{n}\right)}\right]=\frac{\mathbb{E}\left[\max \left\{D_{1}, D_{2}\right\}\right]}{\mathbb{E}\left[D_{1}\right]}
$$

Next, we consider the specific situation of $n$ vertices in $\mathbb{R}^{1}$ with transmit power threshold $D_{i} \sim U_{i}^{p}$, with $U_{i} \sim U[0,1]$, where $U[0,1]$ denotes the uniform distribution, and $p$ models the distance-power gradient.

Corrollary 2. If in the situation of Theorem 1, we have for $i=1, \ldots, n, D_{i} \sim U_{i}^{p}$, with $U_{i} \sim U[0,1]$ then

$$
\frac{W\left(P_{n}\right)}{W\left(T_{n}\right)} \stackrel{a . s}{\longrightarrow} 2-\frac{2}{p+2} .
$$

Proof. We have: $\mathbb{E}\left[D_{1}\right]=\frac{1}{p+1}$, and $\mathbb{E}\left[\max \left\{D_{1}, D_{2}\right\}\right]=$ $\frac{2}{p+2}$.

\section{COMPLETE GRAPH, UNIFORM WEIGHTS}

Often there is no simple relation between power and distance, due to obstacles, reflections and interferences. In the extreme case, the power needed for a successful transmission is fully unrelated to the node positions. In this section, we consider a stylized version of this situation, namely the case where we have a complete graph $G=(V, E, \tilde{X})$ with uniformly distributed edge weights. So $V=\{1,2, \ldots, n\}$ and $\tilde{X}=\left\{X_{i j}: 1 \leq i<j \leq n\right\}$ is a sample of size $\left(\begin{array}{l}n \\ 2\end{array}\right)$ with $X_{i j} \sim U[0,1]$, denoting the edge weights.

As before, we denote with $T_{n}=T_{n}(G)$ a minimum spanning tree of $G$. Let $Y_{n}=Y_{n}(G)$ denote the set of $\lceil n / 2\rceil$ most expensive, and $U_{n}=U_{n}(G)$ the set of $\lfloor n / 2\rfloor$ least expensive edges of $T_{n}$, and $W\left(Y_{n}\right), W\left(U_{n}\right)$ the weight of $Y_{n}, U_{n}$. Note that $W\left(Y_{n}\right)=S_{\lceil n / 2\rceil}\left(T_{n}\right)$.

In order to analyze $W\left(Y_{n}\right)$ and $W\left(U_{n}\right)$, we follow the exposition of Frieze's result (Theorem 2) of [2]. Let $X_{(1)} \leq$
$X_{(2)} \leq \ldots \leq X_{\left(\left(\begin{array}{l}n \\ 2\end{array}\right)\right)}$ denote the order statistics of the sample $\tilde{X}=\left(X_{i j}\right)$. With probability 1 we have $X_{(1)}<X_{(2)}<$ $\ldots<X_{\left(\left(\begin{array}{l}n \\ 2\end{array}\right)\right)}$, implying uniqueness of the minimum spanning tree $T_{n}$. Now $\tilde{X}=\left(X_{i j}\right)$ defines a graph process $G_{t}$ in a natural way, in which edges are added over time, where the edge set of $G_{t}, 0 \leq t \leq\left(\begin{array}{c}n \\ 2\end{array}\right)$, is given by the $t$ least expensive edges of $G$ :

$$
\left\{\{i, j\}: X_{i j}=X_{(k)} \text { for some } k \leq t\right\} .
$$

Given $G_{t}$, define the r.v. $\psi(\cdot)$ as:

$$
\psi(k)=\min \left\{t: w\left(G_{t}\right)=n-k\right\}, k=1,2, \ldots, n-1,
$$

where $w\left(G_{t}\right)$ denotes the number of components of $G_{t}$. Then $\psi(1)=1, \psi(2)=2$ and $\psi(n-1)$ is the first time $t$ when the graph $G_{t}$ is connected. Clearly $\psi(k) \geq k$. By the greedy algorithm of Kruskal, $T_{n}$ is formed by the edges of $G_{t}$ appearing at times $\psi(1), \psi(2), \ldots, \psi(n-1)$, i.e.,

$$
W\left(T_{n}\right)=\sum_{k=1}^{n-1} X_{(\psi(k))} .
$$

By a theorem of Frieze [6] we have:

Theorem 2 ([6]). Let $G=(V, E, \tilde{X})$ be a complete graph, with uniformly distributed edge weights. Then the weight of the minimum spanning tree $T_{n}$ satisfies,

$$
W\left(T_{n}\right) \stackrel{P}{\rightarrow} \zeta(3),
$$

where $\zeta(\cdot)$ denotes the Riemann zeta function.

With $W\left(U_{n}\right)=\sum_{k=1}^{\lfloor n / 2\rfloor} X_{(\psi(k))}$, we show:

Lemma 2. Let $G=(V, E, \tilde{X})$ be a complete graph, with uniformly distributed edge weights. Then :

$$
W\left(U_{n}\right) \stackrel{P}{\rightarrow} 1 / 4 .
$$

Proof. The idea behind the proof of statement (9) is that, for large $n$ and $1 \leq k \leq n / 2, \psi(k)$ is 'close' to $k$. In other words: the least expensive edges of large graphs with uniformly distributed edge weights do not contain a circuit. First we show $\lim _{n \rightarrow \infty} \mathbb{E}\left[W\left(U_{n}\right)\right]=1 / 4$, and then we show convergence in probability. Clearly, as $\mathbb{E}\left[X_{(i)}\right]=i /\left(\left(\begin{array}{l}n \\ 2\end{array}\right)+1\right)$, we have

$$
\left.\mathbb{E}\left[W\left(U_{n}\right)\right]=\sum_{k=1}^{\lfloor n / 2\rfloor} \mathbb{E}[\psi(k)] /\left(\begin{array}{l}
n \\
2
\end{array}\right)+1\right) .
$$

Since $\psi(k) \geq k$, one easily finds $\mathbb{E}\left[W\left(U_{n}\right)\right] \geq 1 / 4$. We will show equality.

First we note:

$$
\mathbb{P}\left(k \leq \psi(k) \leq k+n^{8 / 9}\right)=1-o\left(n^{-1 / 4}\right) .
$$

We show (10) by combining the inequality $\psi(k) \geq k$ with (6.18) in [2] which states that, with probability $1-o\left(n^{-1 / 4}\right)$, $\psi_{0}(k)-n^{8 / 9} \leq \psi(k) \leq \psi_{0}(k)+n^{8 / 9}$, for $k=1, \ldots,\lfloor n / 2\rfloor$, where $\psi_{0}(k)$ is defined by:

$$
u\left(\frac{2 \psi_{0}(k)}{n}\right)=1-\frac{k}{n}
$$


where

$$
u(x)=\sum_{k=1}^{\infty} \frac{k^{k-2}}{k !} x^{k-1} e^{-k x} .
$$

Now, from the fact that $u$ is a one-to-one mapping from $\mathbb{R}^{+}$ to $(0,1]$, and (see [2], page 109) the fact that for $0 \leq x \leq 1$ : $u(x)=1-x / 2$ it follows that: $\psi_{0}(k)=k$, for $0 \leq \bar{k} \leq \bar{n} / 2$, which shows (10).

Next, from (10) and (6.14) in [2] which states, $\mathbb{P}(\psi(n-1) \leq$ $2 n \log n)=1-O\left(n^{-3}\right)$, it follows that

$$
\begin{aligned}
k \leq \mathbb{E}[\psi(k)] & \leq\left(k+n^{8 / 9}\right) \mathbb{P}\left(k \leq \psi(k) \leq k+n^{8 / 9}\right) \\
& +2 n \log n \mathbb{P}\left(k+n^{8 / 9} \leq \psi(k) \leq 2 n \log n\right) \\
& +\left(\begin{array}{l}
n \\
2
\end{array}\right) \mathbb{P}\left(2 n \log n \leq \psi(k) \leq\left(\begin{array}{l}
n \\
2
\end{array}\right)\right),
\end{aligned}
$$

which implies $k \leq \mathbb{E}[\psi(k)] \leq k+n^{8 / 9}+o\left(n^{3 / 4} \log n\right)+$ $O\left(n^{-1}\right)$, whence,

$$
\lim _{n \rightarrow \infty} \mathbb{E}\left[W\left(U_{n}\right)\right]=\lim _{n \rightarrow \infty} \frac{1}{\left(\begin{array}{l}
n \\
2
\end{array}\right)+1} \sum_{k=1}^{\lfloor n / 2\rfloor} \mathbb{E}[\psi(k)]=\frac{1}{4},
$$

completing the first step of the proof. Next, to see convergence in probability, we remark that in [6] equation (15) it is shown that for $1 \leq k \leq \ell \leq n / 2$ :

$$
\mathbb{E}\left[X_{(\psi(k))} X_{(\psi(\ell))}\right]=\frac{\mathbb{E}[\psi(k) \psi(\ell)]+\mathbb{E}[\psi(k)]}{\left(\left(\begin{array}{l}
n \\
2
\end{array}\right)+1\right)\left(\left(\begin{array}{l}
n \\
2
\end{array}\right)+2\right)},
$$

so in order to show that $\operatorname{Var}\left[W\left(U_{n}\right)\right] \rightarrow 0$ it suffices to show that

$$
\sum_{k=1}^{\lfloor n / 2\rfloor} \sum_{\ell=1}^{\lfloor n / 2\rfloor} \mathbb{E}[\psi(k) \psi(\ell)] \leq(1+o(1)) \sum_{k=1}^{\lfloor n / 2\rfloor} \sum_{\ell=1}^{\lfloor n / 2\rfloor} \mathbb{E}[\psi(k)] \mathbb{E}[\psi(\ell)]
$$

This follows from [6] equation (17) which implies that for some constant $c$, and $1 \leq k \leq \ell \leq n / 2$ :

$$
\mathbb{E}[\psi(k) \psi(\ell)] \leq \mathbb{E}[\psi(k)] \mathbb{E}[\psi(\ell)]+c n^{11 / 6}(\log n)^{2}
$$

Now, we obtain (9) from Chebyshev's inequality.

This leads to the following theorem for power assignments.

TheOrem 3. Let $G=(V, E, \tilde{X})$ be a complete graph, with uniformly distributed edge weights. Then,

$$
\limsup _{n \rightarrow \infty} \mathbb{E}\left[\frac{W\left(P_{n}\right)}{W\left(T_{n}\right)}\right] \leq \frac{2(\zeta(3)-1 / 4)}{\zeta(3)} \approx 1.58 \ldots
$$

Proof. As $W\left(T_{n}\right) \leq W\left(P_{n}\right) \leq 2 W\left(Y_{n}\right)$, and $W\left(T_{n}\right)=$ $W\left(U_{n}\right)+W\left(Y_{n}\right)$, it follows from Theorem 2 and Lemma 2 that $\zeta(3) \leq \lim \sup _{n \rightarrow \infty} \mathbb{E}\left[W\left(P_{n}\right)\right] \leq 2(\zeta(3)-1 / 4)$.

\section{CONCLUDING REMARKS}

Worst-case, the approximation of the MST heuristic for the power assignment problem can be strengthened to two times the weight of the heavier half of the edges (instead of two times the whole MST). Moreover, in the one-dimensional case, with uniform distributed distances between neighboring vertices, and a distance power gradient of $p$, the expected approximation ratio is bounded above by $2-2 /(p+2)$. For the complete graph with uniform $[0,1]$-distributed edge weights, the expected approximation ratio is asymptotically bounded above by $2-1 / 2 \zeta(3) \approx 1.58 \ldots$. Heuristics as presented in [1] are interesting for further analysis.

\section{ACKNOWLEDGEMENTS}

This work was performed in the project RRR (Realisation of Reliable and Secure Residential Sensor Platforms), IOP Generieke communicatie, IGC1020.

\section{REFERENCES}

[1] Althaus, E., Calinescu, G., Mandoiu, I.I. Et.Al. . (2006). Power efficient range assignment for symmetric connectivity in static ad hoc wireless networks, Wireless Networks, 12, 287-299.

[2] Bollobas, B. (2001). Random Graphs, 2nd edition, Cambridge University Press, ISBN 052179722.

[3] Caragiannis, I., Flammini, M., and Moscardelli, L. (2013) An exponential improvement to the MST heuristic for minimum energy broadcasting in ad hoc wireless networks, IEEE/ACM Transactions on Networking, 21(4), 1322-1331.

[4] Chen, W. and Huan, N. (1989). The strongly connecting problem on multihop packet radio networks, IEEE Transactions on Communications, 37, 293-295.

[5] Clementi, A.E.F, Penna, P. And Silvestri, R. (2000). On the power assignment problem in radio networks, Electronic Colloquium on Computational Complexity, Report No. 54.

[6] Frieze, A.M. (1985). On the value of a random minimum spanning tree problem. Discrete Applied Mathematics, 10, 47-56.

[7] Fuchs, B. (2006). On the hardness of range assignment problems, Algorithms and Complexity, Lecture Notes in Computer Science, Springer Berlin, Heidelberg, 3998, 127-138.

[8] De Graaf, M, Boucherie, R.J., van Ommeren, J.C.W. AND HuRINK, J.L. (2007) An average case analysis of the minimum spanning tree heuristic for the range assignment problem, Research report 1857, University of Twente.

[9] De Graaf, M. and Manthey, B. (2014) Probabilistic analysis of power assignments. In: 39th International Symposium on Mathematical Foundations of Computer Science (MFCS 2014), 25-29 Sep 2014, Budapest, Hungary. pp. 201-212. Lecture Notes in Computer Science $\mathbf{8 6 3 5}$.

[10] Lloyd, E., Liu, R., Marathe, M., Ramanathan, R., Ravi, S. (2005). Algorithmic Aspects of Topology Control problems for ad-hoc networks, Mobile Networks and applications, 10, Issue 1-2, 19-34.

[11] Rossi, G.V. And Leung, K.K. (2013) Performance tradeoffs by power control in wireless ad-hoc networks, in: The 9th International Wireless Communications $\mathcal{E}$ Mobile Computing Conference (IWGCMC 2013), 1343-1347.

[12] Santi, P. Blough, D., Vainstein, F. (2001). A probabilistic analysis for the range assignment Problem in ad-hoc networks, in: MobiHoc '01: Proceedings of the 2nd ACM International symposium on Mobile ad hoc networking $\&$ computing, ACM Press, New York, 212-220. 